\title{
Interactive comment on "Unshielded precipitation gauge collection efficiency with wind speed and hydrometeor fall velocity. Part I: modelling results" by Jeffery Hoover et al.
}

\section{Anonymous Referee \#2}

Received and published: 5 January 2021

In this work the authors presented "A new method for assessing collection efficiency using wind speed and hydrometeor fall velocity", but this methodology, based on CFD simulations and Lagrangian particle tracking model have been previously used in the recent literature (e.g. Tériault et al. 2012, Colli et al. 2016a,b). The Geonor precipitation gauge has been studied in these works in both shielded and unshielded configuration.

One of the main conclusion of this work is the relation between "Collection Efficiency" (CE) and the particle fall velocity instead of the particle diameter as shown in Colli et al. 
2016b. However, for wet and dry snow they use the relation proposed by Rasmussen et al. 1999 to calculate the particle fall velocity as a function of the particle diameter. Furthermore, in equation 9 and 17 the authors reported the formulas for the "overall Collection Efficiency" for rain and snow respectively. In these equations is highlighted that the fall velocity is a function of the particle diameter (D) and therefore the overall CE depends only on the wind speed and D. For this reason, there is no novelty in this approach.

L 175: The authors use the relation proposed by Rasmussen et al. 1999 to calculate the terminal velocity, and they stated that "hydrometeor density was chosen to provide the desired hydrometeor fall velocity", but in the work of Rasmussen et al. the density value relations are provided for both wet and dry snow. How did the authors vary the hydrometeor density? Are these density values realistic? or are they used only to obtain the fall velocity the authors desired? The smaller particle of wet snow has a density value greater than water, is it right?

Fig. 5 and 6 : in figure 5 the authors showed the "collection efficiency" for different precipitation types and fall velocities respect to wind speed. It is clear from the figure that there are differences in the CE values of different precipitation type but with the same fall velocity. Furthermore, the authors used only part of these data to obtain the "empirical collection efficiency expression" showed in figure 6, but this relation has been used to calculate the "overall Collection Efficiency" for all the particle types. How do this affect the obtained results?

Sections 3.4.3 and 4.43: in these sections (Results and Discussion sections) the authors highlight the dependency of overall CE with precipitation intensity. This topic is addressed in the recent work of Colli et al. 2020. Do the authors compare their results with that work?

Printer-friendly version

Discussion paper 
In general, in this work the authors reproduced methodologies used in previous works and there are no significant improvements or novelty. Furthermore, there are a few points the authors need to clarify, like e.g. the choice of the particle density values and the use of an unique empirical CE relation for different precipitation types and they need to evaluate how these impact on the results.

Interactive

comment

Reference:

Colli, M., Stagnaro, M., Lanza, L. G., Rasmussen, R. and Thériault, J. M. (2020). Adjustments for wind-induced undercatch in snowfall measurements based on precipitation intensity, Journal of hydrometeorology, 21, 1039-1050.

Interactive comment on Hydrol. Earth Syst. Sci. Discuss., https://doi.org/10.5194/hess-2020553, 2020. 